\title{
Investigations of the Simian Ontogenic Switch from Fetal to Adult Hemoglobin at the Progenitor Cell Level
}

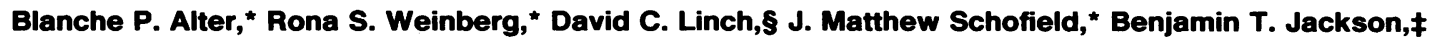 \\ George J. Piasecki,‡ John C. Thornton, ${ }^{\star}$ and David G. Nathan \\ *Polly Annenberg Levee Hematology Center, Departments of Medicine, Pediatrics, and Biomathematical Sciences, Mount Sinai School of \\ Medicine, New York, New York 10029; ¥Surgical Service, Providence Veterans Administration Medical Center, and Department \\ of Surgery, Program in Medicine, Brown University, Providence, Rhode Island 02912; \$Division of Hematology/Oncology, \\ Children's Hospital Medical Center and Dana-Farber Cancer Institute, and Department of Pediatrics, \\ Harvard Medical School, Boston, Massachusetts 02115
}

\begin{abstract}
The ontogenic switch from fetal to adult hemoglobin could result from discontinuous events, such as replacement of fetal erythroid progenitor cells by adult ones, or gradual modulation of the hemoglobin program of a single progenitor cell pool. The former would result in progenitors at midswitch with skewed fractional $\beta$-globin synthesis programs, the latter in a Gaussian distribution. For these studies, we obtained bone marrow from rhesus monkey fetuses at 141-153 d (midswitch). Mononuclear cells were cultured in methyl cellulose with erythropoietin, and single BFUE-derived colonies were removed and incubated with $\left[{ }^{3} \mathbf{H} \mid\right.$ leucine. Globin synthesis was examined by gel electrophoresis and fluorography. The $\beta$-globin synthesis pattern of single fetal colonies was skewed, and did not fit a normal distribution. The fetal pattern resembled the pattern of an artificial mixture of fetal and adult progenitors, suggesting that the fetal progenitor pool could contain populations with different $\beta$-globin programs. This nonGaussian distribution in the progenitors of midswitch fetuses is consistent with a discontinuous model for hemoglobin switching during ontogeny.
\end{abstract}

\section{Introduction}

The switch from fetal to adult hemoglobin that occcurs during ontogeny is regulated such that a progressive accumulation of erythrocytes with increasing content of adult hemoglobin replaces fetal cells that contain $>90 \%$ fetal hemoglobin. Whether the switch is characterized by a series of intermittent steps or functions is currently unknown (1).

In recent years it has been possible to examine the fetal switch at the level of erythroid precursors and even progenitors by measurement of hemoglobin synthesis in freshly obtained erythroblasts, erythroblasts derived from human fetal or newborn blood, or simian marrow erythroid progenitors driven to differentiate

This work was presented in part at the American Society of Hematology, 3 December 1984 in Miami, FL, and was published in abstract form (Blood. 1984. 64[Suppl. 1]:60A).

Dr. Linch's current address is Department of Haematology, The Middlesex Hospital, London, England.

Address reprint requests to Dr. Blanche P. Alter, Division of Hematology, Mount Sinai School of Medicine, 1 Gustave L. Levy Place, New York, NY 10029. 1986.

Received for publication 11 June 1985 and in revised form 25 August

The Journal of Clinical Investigation, Inc.

Volume 78, December 1986, 1497-1503 in vitro (2-16). These studies have demonstrated that the forward switch is in fact programmed at the progenitor level. Before the onset of the switch, erythroid progenitors produce erythroblasts that synthesize fetal hemoglobin almost exclusively. Just at the onset of the switch, when marrow erythroblasts still exhibit very little adult hemoglobin synthesis, progenitor-derived erythroblasts have clearly begun to switch to the adult phenotype. Thus, the change in the phenotype of the progenitor-derived erythroblast is the harbinger of an event that directs the future phenotype of recognizable marrow precursor cells. After this early point in the switch the phenotype of progenitor-derived (particularly erythroid burst forming unit) erythroblasts becomes unexpectedly weighted toward hemoglobin $\mathrm{F}(\mathrm{Hb} \mathrm{F})^{1}$ production. At midswitch, erythroblasts derived from progenitors in vitro synthesize much more $\mathrm{Hb} F$ than erythroblasts that populate the same marrow in vivo. This peculiar hemoglobin phenotype of progenitor-derived erythroblasts persists through adult simian life. The same phenomenon is observed in humans as well, but at a much lower level of $\mathrm{Hb} F$ production (17).

We and others have previously demonstrated that all early erythroid progenitors in adult humans or simians produce colonies of erythroblasts that contain some $\operatorname{Hb} F(7,8,10,11)$. The fractional synthesis of $\mathrm{Hb} \mathrm{F}$ per colony exhibits a normally distributed (Gaussian) pattern $(7,11)$. Published data regarding the human ontogenic switch have suggested that the fractional synthesis of $\mathrm{Hb} \mathrm{F}$ is distributed similarly to that of the adult, but this conclusion was in fact derived from analysis of small numbers of colonies, insufficient to fully discriminte between discrete vs. continuous models for the switch $(1,6-9)$.

The purpose of the studies described in this report was to examine the characteristics of the fetal-to-adult hemoglobin switch to determine whether the phenotype of erythroid progenitors shifts toward adult hemoglobin synthesis so gradually that the fraction of $\mathrm{Hb} \mathrm{F}$ production in individual colonies exhibits Gaussian characteristics, or whether a residual population of progenitors programmed for high $\mathrm{Hb} \mathrm{F}$ synthesis coexists with adult programmed cells, particularly at midswitch when the two discrete populations might be expected to be detectable. A gradual or continuous evolution of the $\mathrm{Hb} F$ program within a single clone would retain the Gaussian distribution pattern. In contrast, a discrete, discontinuous ontogenic switch, due to clonal replacement or the intermittent effect of environmental factors, would lead to a skewed and possibly bimodal distribution.

Previous studies of switching at the progenitor level were carried out with human cord blood (6-11). Although our own

1. Abbreviations used in this paper: $\mathrm{Hb} \mathrm{A}$, hemoglobin $\mathrm{A} ; \mathrm{Hb} \mathrm{F}$, hemoglobin F. 


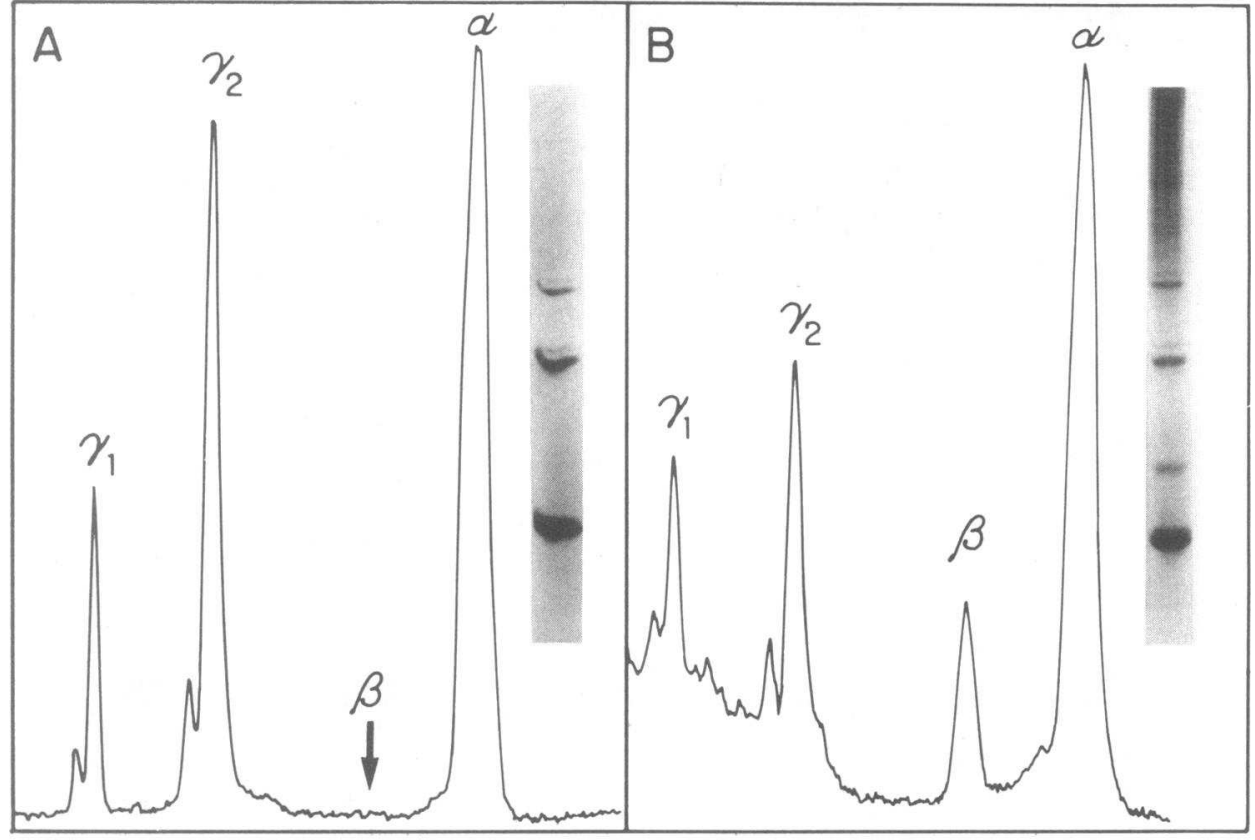

(t)

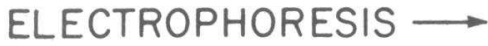

Figure 1. Globin synthesis in rhesus fetal BFU-E-derived colonies. The fetus was $151 \mathrm{~d}$ gestation. The two colonies are from the same culture plate and were labeled from day 8 to 9. Insets show fluorograms of gel lanes from $\left[{ }^{3} \mathrm{H}\right]$ leucine-labeled colonies. Figures show densitometric tracings. $(A) \beta, 1 \%$. (B) $\beta, 30 \%$. experiments suggested the presence of progenitors with discrete hemoglobin programs $(10,11)$, definitive studies were not possible. In man, the middle of the switch from $\mathrm{Hb} \mathrm{F}$ to hemoglobin A (Hb A) occurs at $\sim 1$ mo of postnatal age $(4,5)$, a time not readily accessible to study. We therefore chose to use a simian model, in which midswitch occurs at $140-160 \mathrm{~d}$ in utero (12). Our results suggest that a mixture of discrete populations of erythroid progenitor cells may coexist during the midswitch period, because the pattern of fractional $\mathrm{Hb} F$ synthesis in single colonies is non-Gaussian.

\section{Methods}

Animals. Rhesus monkeys (Macaca mulatta) were cared for at the New England Regional Primate Research Center in Southborough, MA, and all procedures were approved by the scientific advisory committee at the Center. Bone marrow was obtained from the femurs of fetuses from timed pregnancies, delivered by cesarean section. The femurs were dissected out sterilely, the ends snipped off, and the marrow flushed out with $\alpha$-medium. The four fetuses were of 141, 143, 151, and $153 \mathrm{~d}$ gestation, within the range that we have previously shown to embrace the midswitch period (12). Adult marrow was obtained by aspiration of the iliac crests of the anesthetized mothers at the time of cesarean section. These procedures have been described previously (12).

Cell culture. Bone marrow mononuclear cells were isolated by centrifugation on Ficoll-Hypaque and either used directly or slowly cryopreserved and later thawed (18). Mononuclear cells from either source were washed and suspended in $\alpha$-medium, and then plated in methyl cellulose at 100,000 cells/ml (final concentration) using methods similar to those described for human cultures (10). Human urinary erythropoietin, kindly provided by the Division of Blood Diseases and Resources of the National Heart, Lung, and Blood Institute, was used at 1-2 U/ $\mathrm{ml}$. Each culture was in a final volume of $0.3 \mathrm{ml}$, and colony counts were determined from three culture plates. The peak growth of colonies was between days 8 and 10. Each study of individual colonies represents all of the recognizable erythroid colonies in one or more culture plates.

Globin synthesis. Individual colonies were incubated for 16-24 h with $\left[{ }^{3} \mathrm{H}\right]$ leucine and harvested in the presence of nonradioactive carrier fetal and adult erythrocytes that had been previously cryopreserved in glycerol. Globin chains were separated by electrophoresis on Triton acid urea polyacrylamide gels, and relative radioactivity determined by fluorography, followed by planimetry of $\mathrm{x}$-rays scanned in a densitometer (Gilford Instrument Laboratories, Inc., Oberlin, $\mathrm{OH}$ ). Details of all of these methods are provided elsewhere $(10,12)$. Percentage of $\beta$ synthesis was calculated as $100 \times \beta /\left(\gamma_{1}+\gamma_{2}+\beta\right)$.
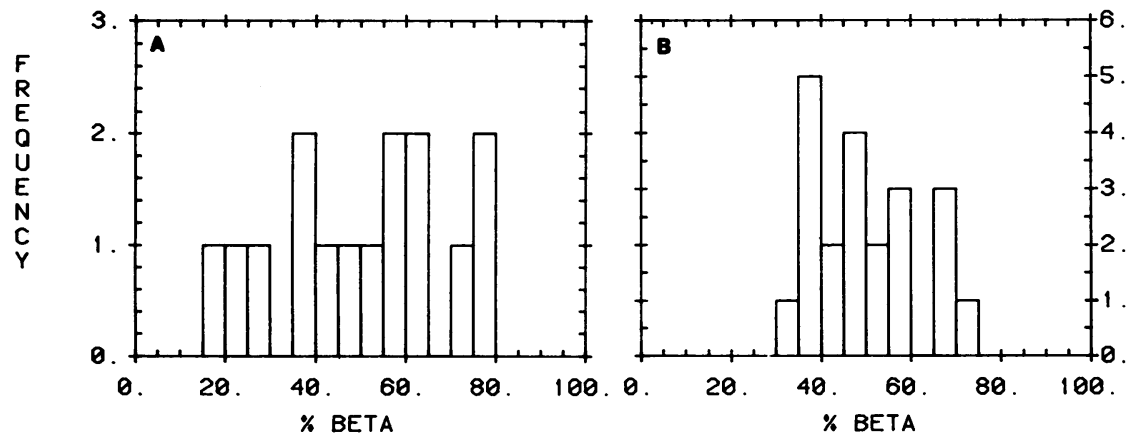

Figure 2. Histograms of $\beta$-globin synthesis in adult rhesus bone marrow BFUE-derived colonies. $A$ and $B$ are from two separate studies. $(A)$ Labeled day 8 to 9. (B) Labeled day 10 to 11. 


\begin{tabular}{|c|c|c|c|c|c|c|c|c|c|c|c|}
\hline \multirow[b]{2}{*}{ Sample } & \multirow[b]{2}{*}{$n$} & \multirow[b]{2}{*}{$\beta$ mean } & \multirow[b]{2}{*}{$\beta$ median } & \multirow[b]{2}{*}{$\beta$ range } & \multirow[b]{2}{*}{$\beta \mathrm{SD}$} & \multicolumn{6}{|c|}{ Tests for normality } \\
\hline & & & & & & $\begin{array}{l}\text { Wilk-Shapiro } \\
\text { statistic }\end{array}$ & $P$ & Skew & $P$ & $\begin{array}{l}\text { Kolmogorov-Smirnov } \\
\text { statistic }\end{array}$ & $P$ \\
\hline All & 15 & 50.3 & 53.2 & $19.7-78.3$ & 18.5 & 0.96 & $>0.05$ & 0.23 & $>0.05$ & 0.06 & $>0.05$ \\
\hline All & 21 & 49.7 & 47.0 & $30.2-72.3$ & 12.4 & 0.94 & $>0.05$ & 0.44 & $>0.05$ & 0.12 & $>0.05$ \\
\hline
\end{tabular}

Statistics and graphics. Data were analyzed and graphs plotted with PROPHET, a timesharing computer resource sponsored by the Division of Research Resources of the National Institutes of Health. The tests for normal distribution were Wilk-Shapiro, when $n \leq 50$, and D'Agostino's, when $n>50$, as well as the Kolmogorov-Smirnov test of goodness of fit to a normal distribution (19). Cumulative relative frequency plots were also examined (20). The level of significance for all statistical tests was 0.05 .

\section{Results}

Fetal bone marrow erythroid colony growth, derived from BFU$E$, was maximal on days 8-10 and required $<1 \mathrm{U} / \mathrm{ml}$ of erythropoietin. The number of colonies was usually between 50 and 300 per 100,000 plated cells. The appearance of the rhesus colonies resembled those seen in human cultures, although they were somewhat smaller than human colonies, in part due to the smaller size of rhesus cells. Adult rhesus colonies also achieved maximal growth on days 8-10, although they were usually smaller and not as red as the fetal colonies.

Globin synthesis in two representative fetal colonies is shown in Fig. 1. The colonies shown are from a single culture plate and indicate the extremes of the range of globin synthetic patterns. The proportion of non- $\alpha$-synthesis that was $\beta$ was $1 \%$ and $30 \%$ in these two colonies, and the differences in the level of radioactivity in the $\beta$-bands are clear.

Individual BFU-E-derived colonies from adult bone marrow are shown in Fig. 2. The means and medians for $\beta$-synthesis were $\sim 50 \%$, and the histograms were consistent with a normal distribution by all tests. The statistics are shown in Table I. The ranges of $\beta$-synthesis were $19.7-78.3 \%$ in one study and $30.2-$ $72.3 \%$ in the other. These are within the range of $\beta$-synthesis seen previously in adult marrows (12). Further analysis of the patterns of $\beta$-globin synthesis was performed with cumulative relative frequency distribution plots (Fig. 3). In each of the two studies the data form a straight line, consistent with normal distributions.

To determine the pattern of $\beta$-synthesis resulting from a mixture of two clearly discrete populations, the experiment depicted in Fig. 4 was performed. Erythroid colonies were grown simultaneously from the marrow of a 153-d fetus and its mother. In addition, co-cultures were established with a mixture of equal numbers of fetal and adult mononuclear cells $(100,000$ of each per milliliter). More than 40 colonies were included in each analysis.

The separate cultures result in patterns of fractional $\beta$-synthesis that have two nonoverlapping distributions (Fig. $4 A$ ). The co-culture generated a $\beta$-synthesis pattern that was not quite as discrete but appeared to have a similar pattern (Fig. $4 \mathrm{~B}$ ). In both circumstances, the mean $\beta$-synthesis was $25 \%$ (Table II). In experiments where fetal and adult marrow were cultured separately but the data were analyzed collectively, the Wilk-Shapiro and Kolmogorov-Smirnov tests were both consistent with a pattern that was not distributed normally, as was expected for two distinct populations. In the co-culture, the Wilk-Shapiro test also rejected the hypothesis that the data were normally distributed. There was instead a significant skew. The cumulative relative frequency distribution plots shown in Fig. 5 demonstrate the patterns seen when there are two populations (20). The left sides of the plots show downward curves, with data lying above the straight line derived by linear regression analysis. The middle of the curve crosses the straight line and then returns to meet the line.

The patterns of $\beta$-synthesis by individual fetal BFU-E-derived colonies are shown in Figs. 6 and 7, and analyzed in Table III. All of the fetal $\beta$-histograms were positively skewed and not

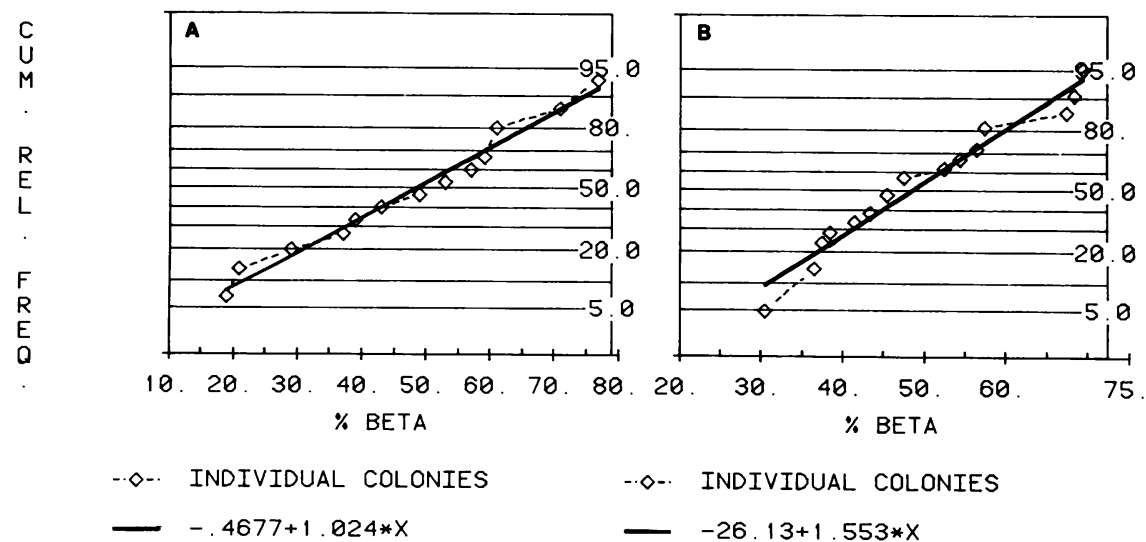

Figure 3. Cumulative relative frequency distributions for the adult $\beta$-globin synthesis data in Fig. 2 . The ordinate is a normal probability scale. The solid line shows the best fit by linear regression. The dashed line connects the data points, shown as midpoints of cumulative intervals. 

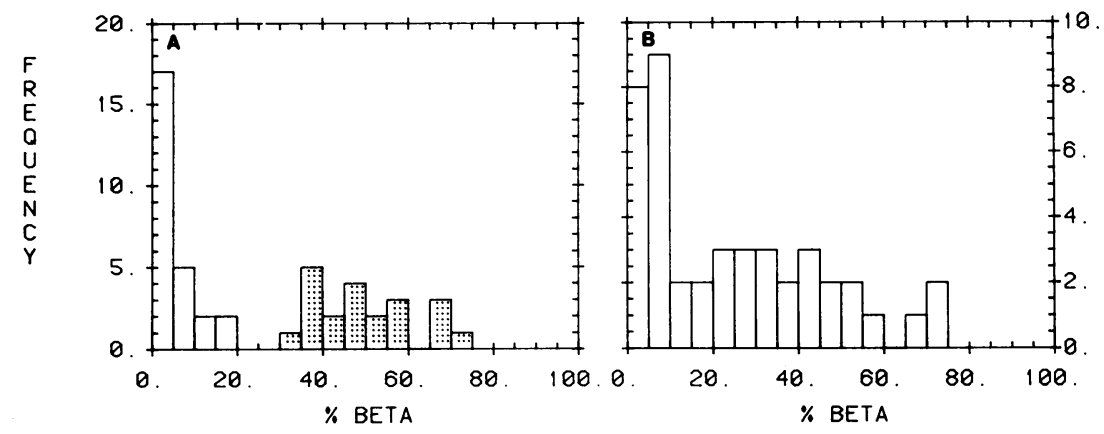

Figure 4. Histograms of $\beta$-synthesis in rhesus marrow BFU-E-derived colonies. (A) 153-d fetus and mother, cultured separately and plotted together. Dots show data from mother's culture. $(B)$ Fetus and mother co-cultured. normally distributed. The only exception is the analysis of the 153-d fetal samples by D'Agostino's test, in which $P$ is between 0.05 and 0.1 . However, this sample appears bimodal in shape (Fig. $6 C$ ), and $P<0.05$, according to the Kolmogorov-Smirnov test of goodness of fit. The pattern in Fig. $6 C$ has two possible modes, at $1-2 \%$ and $25-26 \% \beta$-globin. In all studies, the mean percentage of $\beta$-synthesis exceeds the median, consistent with the positive skew. For each fetal study, $\beta$-synthesis data were arbitrarily grouped into those with "low" and "high" $\beta$-synthesis, with ranges determined from the histograms. These arbitrary groupings usually resulted in two separate populations in which the skew was less evident (Table III). The cumulative relative frequency plots shown in Fig. 7 resemble those shown in Fig. 5. They all curve downward on the left, cross the straight line, and achieve a new slope, compatible with the interpretation of bimodality. Therefore, both statistical and graphic analyses of the $\beta$-synthesis patterns in the four studies of fetal marrow indicate that they are clearly different from the patterns observed in identical studies of adult marrow. The fetal studies closely resemble the patterns that result from two clearly discrete populations such as those observed when fetal and maternal samples are mixed (Figs. 4 and 5).

\section{Discussion}

The experiments described in this report were designed to compare the distributions of $\mathrm{Hb} \mathrm{F}$ programs expressed in erythroid colonies derived from marrow erythroid progenitors at midswitch with those present in adult life. From such experiments we hoped to determine whether the fetal-to-adult switch in simians can be represented as a gradual and smoothly running biological clock, or whether the transition from fetal-to-adult hemoglobin synthesis is actually discontinuous. In the former model, fractional $\beta$-globin synthesis in individual progenitor-derived erythroid colonies would ideally conform to a Gaussian distribution, as it does in adult marrow cultures. In contrast, an intermittent switch would result in at least two populations of progenitors, those that give rise to colonies with distributions of $\beta$ - and $\gamma$-globin synthesis characteristic of early fetuses and those with a distribution of globin synthesis observed in the colonies derived from adult marrow progenitors. Clearly, the two possible results would suggest different regulatory models of $\mathrm{Hb}$ F-to-Hb A switching at the progenitor level. A Gaussian distribution at midswitch would suggest that the switch mechanism operates randomly in progenitors and gradually moves forward in all of them so that the kinetics can be described as a series of normal curves with progressively increasing mean $\beta$-synthesis levels.

In contrast, a discontinuous model, in which discrete populations of progenitors with adult and fetal programs are present, would lead to a skewed, non-Gaussian pattern. This would best be explained by one of two mechanisms: a clonal replacement model in which fetal progenitors are progressively replaced by adult progenitors, or by intermittent activity of a switching factor that might "switch over" the Hb F-to-Hb A program of a subset of fetal progenitors to an adult program at intervals during gestation. The experiments presented here cannot distinguish between these two discontinuous models but suggest that one of them must be operative. Unlike adult marrow, in which the propensity to synthesize $\mathrm{Hb} \mathrm{F}$ in progenitor-derived erythroblasts can be described as a normal distribution, a skewed pattern is detected in fetal marrow. This conclusion was strongly supported

Table II. Percentage $\beta$-Globin Synthesis in Individual Fetal and Adult Erythroid Colonies

\begin{tabular}{|c|c|c|c|c|c|c|c|c|c|c|c|}
\hline \multirow[b]{2}{*}{ Sample } & \multirow[b]{2}{*}{$n$} & \multirow[b]{2}{*}{$\beta$ mean } & \multirow[b]{2}{*}{$\beta$ median } & \multirow[b]{2}{*}{$\beta$ range } & \multirow[b]{2}{*}{$\beta \mathrm{SD}$} & \multicolumn{6}{|c|}{ Tests for normality } \\
\hline & & & & & & $\begin{array}{l}\text { Wilk-Shapiro } \\
\text { statistic }\end{array}$ & $P$ & Skew & $P$ & $\begin{array}{l}\text { Kolmogorov-Smirnov } \\
\text { statistic }\end{array}$ & $P$ \\
\hline \multicolumn{12}{|c|}{ Fetus and mother cultured separately } \\
\hline All & 47 & 25.2 & 14.8 & $1.1-72.3$ & 24.0 & 0.83 & $<0.01$ & 0.53 & $>0.05$ & 0.20 & $<0.05$ \\
\hline$\beta \leq 20$ & 26 & 5.5 & 3.4 & $1.1-18.2$ & 4.7 & 0.77 & $<0.01$ & 1.56 & $<0.05$ & 0.24 & $>0.05$ \\
\hline$\beta>20$ & 21 & 49.7 & 47.0 & $30.2-72.3$ & 12.4 & 0.94 & $>0.05$ & 0.44 & $>0.05$ & 0.12 & $>0.05$ \\
\hline \multicolumn{12}{|c|}{ Fetus and mother co-cultured } \\
\hline All & 43 & 24.8 & 20.3 & $1.7-73.7$ & 21.2 & 0.88 & $<0.01$ & 0.78 & $<0.05$ & 0.17 & $>0.05$ \\
\hline$\beta \leq 20$ & 21 & 6.9 & 6.2 & $1.7-16.3$ & 4.0 & 0.91 & $>0.05$ & 1.10 & $<0.05$ & 0.12 & $>0.05$ \\
\hline$\beta>20$ & 22 & 41.9 & 39.4 & $20.3-73.7$ & 16.0 & 0.94 & $>0.05$ & 0.64 & $>0.05$ & 0.10 & $>0.05$ \\
\hline
\end{tabular}




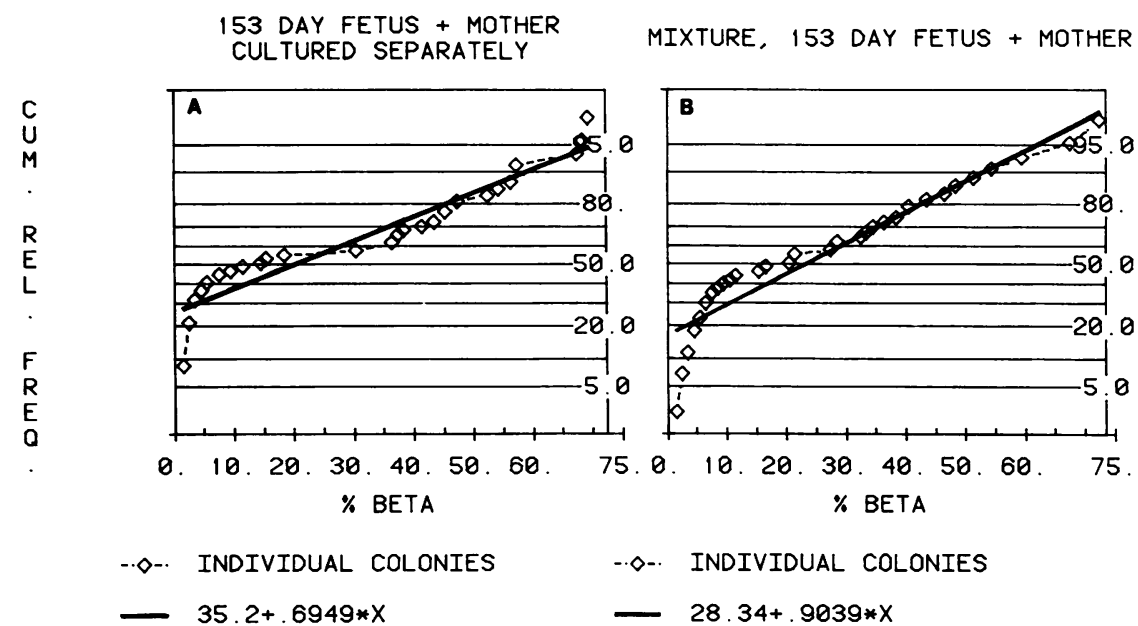

Figure 5. Cumulative relative frequency distributions for the combined fetal and maternal $\beta$-globin synthesis data in Fig. 4. See Fig. 3 legend for explanation. by statistical treatment of data derived from analyses of a large number of colonies in each of several experiments. For this purpose, we examined at least 24 and sometimes more than 50 colonies in each study of four individual rhesus monkey fetuses at midswitch (141-153 d gestation). A similar number of colonies was examined in each of two separate studies of adult marrow.

To demonstrate the pattern expected for a mixed population of fetal progenitors at midswitch, fetal and adult progenitors were examined in two ways. In the first, the mononuclear cells from a fetus and its mother were cultured separately, but the data were pooled for analysis. In the second, the mononuclear cells were co-cultured, and the results analyzed again. The two discrete populations (fetal and maternal) produced $\beta$-synthesis histograms with extreme positive skew and two modes. The two populations had expectedly different distribution widths: narrow for the fetal and wider for the adult colonies.

As emphasized above, the data derived from these assays of fetal marrow cannot be readily explained by a model charac- terized by continuous phenotypic change of a single population. The data are more consistent with discontinuous clonal replacement or the discontinuous effect of an exogenous switching factor. A sheep model has been used by other investigators to examine the possible role of exogenous environmental influences in globin gene regulation (21-24). Hematopoietic progenitor cells from adult sheep transplanted into fetuses continued to produce adult hemoglobin (22), suggesting that the fetal environment is unable to influence the hemoglobin expression program of adult progenitor cells. In contrast, transplantation of fetal progenitor cells into adult sheep has lead to production of adult hemoglobin by those fetal cells $(21,23,24)$, but only if the fetus had already begun to switch in vivo. These observations were interpreted to indicate that the fetal progenitor cell can undergo an environmentally regulated change toward an adult hemoglobin phenotype. Indeed, in these experiments adult hemoglobin appeared more rapidly than when the cells remained within the fetal host. However, an alternative explanation, also consistent with those
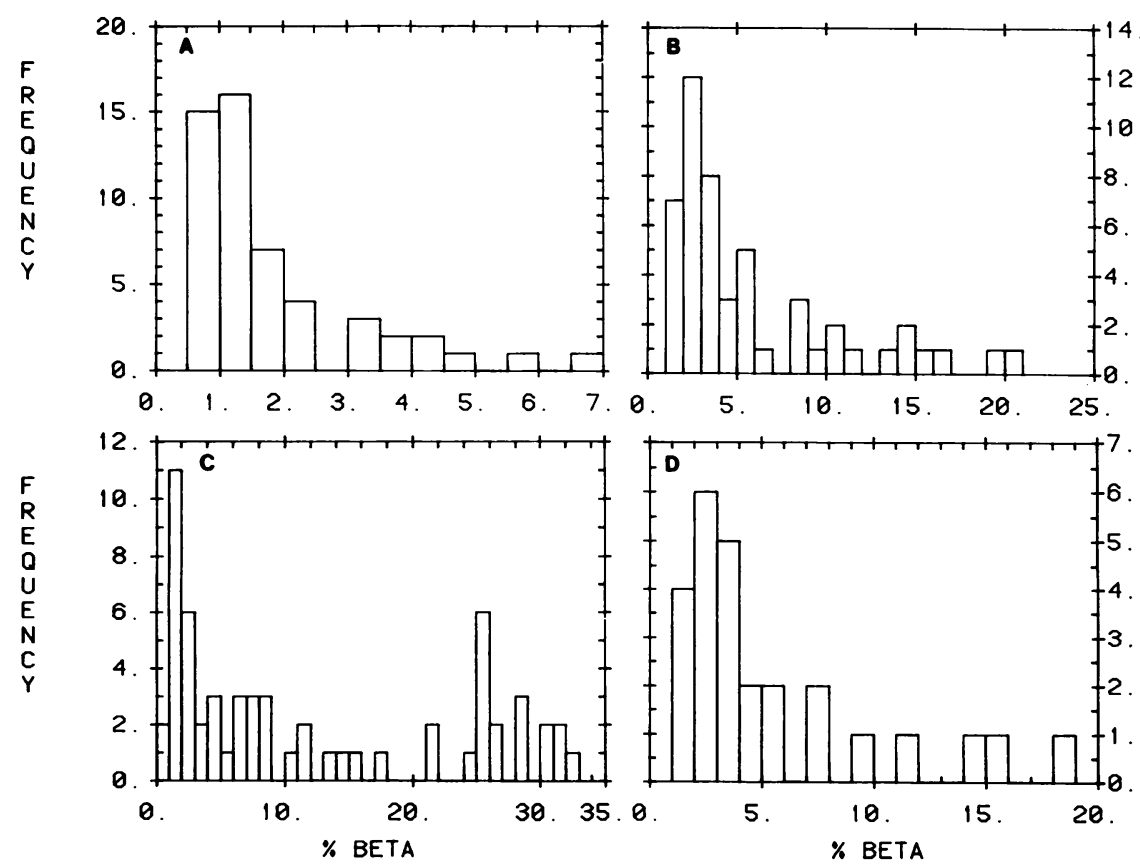

$F$
$R$
$E$
$O$
$U$
$E$
$N$
$C$
$Y$

$F$
$R$
$E$
$O$
$U$
$E$
$N$
$C$
$Y$

Figure 6. Histograms of $\beta$-globin synthesis in rhesus fetal marrow BFU-E-derived colonies. $(A)$ 141-d gestation, labeled day 8 to 9 . (B) 143-d gestation, labeled day 8 to 9 . (C) 151-d gestation, labeled day 8 to 9 . (D) 153-d gestation, labeled day 10 to 11 . 

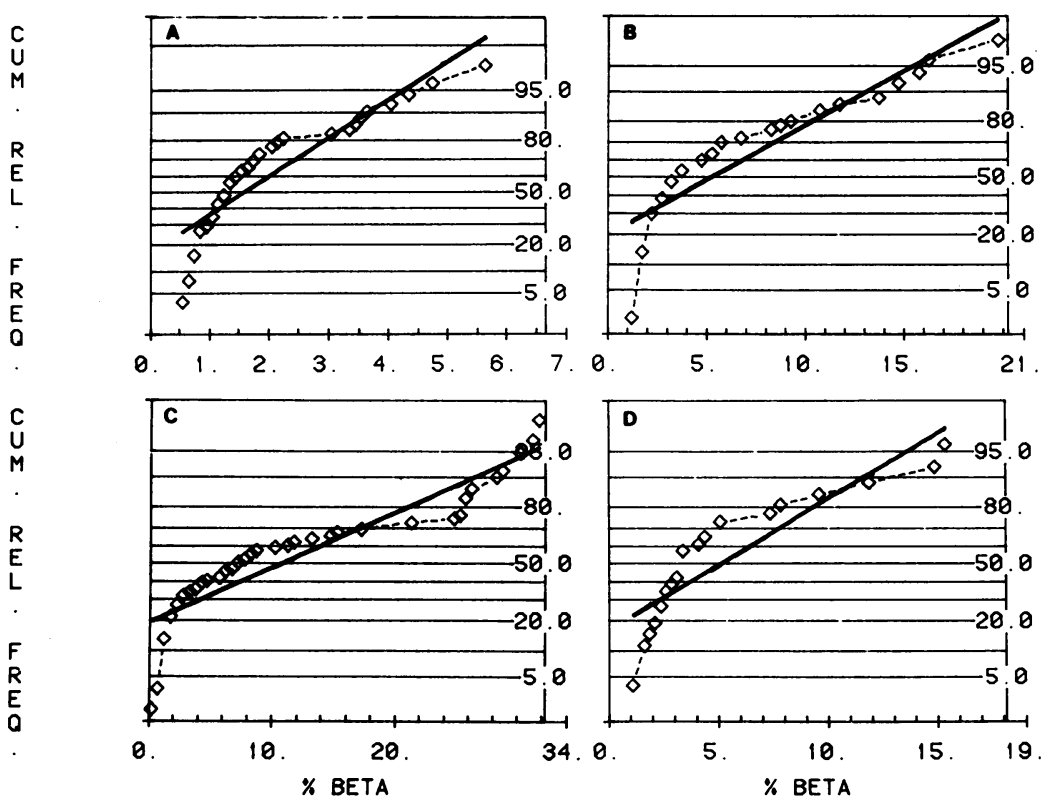

Figure 7. Cumulative relative frequency distributions for fetal $\beta$-globin synthesis data in Fig. 6. See Fig. 3 legend for explanation.

data, is that older fetuses develop a discrete population of adult progenitors that have a growth advantage in the adult host. The only reports of human fetal liver transplants (for leukemia in irradiated children) were with first-trimester fetal material (i.e., at preswitch) $(25,26)$. The results are also consistent with a clonal rather than environmentally influenced model. The progenitors of early fetuses, in the preswitch period, gave rise to colonies of erythroblasts solely capable of $>95 \% \mathrm{Hb} F$ synthesis.

In murine studies, fetal liver erythroid progenitors were found to be very sensitive to low levels of erythropoietin in vivo, whereas adult progenitors were less erythropoietin sensitive (27). Younger embryos had more progenitor cells with high erythropoietin sensitivity than did older embryos, which in turn had more than those found in adult mice. Similarly, in human studies, fetal erythroid progenitors were more sensitive to low levels of erythropoietin than were adult progenitors (2). These observations provide further support for a discrete model of the ontogenic switch.

In conclusion, we believe that the data presented here, together with data previously published by others as well as ourselves, are consistent with a discrete clonal or intermittent environmentally driven model for hemoglobin switching during ontogeny. Though we cannot resolve these options, our present data are entirely inconsistent with a gradual smoothly functioning

Table III. Percentage $\beta$-Globin Synthesis in Individual Fetal Erythroid Colonies

\begin{tabular}{|c|c|c|c|c|c|c|c|c|c|c|c|}
\hline \multirow[b]{2}{*}{ Sample } & \multirow[b]{2}{*}{$n$} & \multirow[b]{2}{*}{$\beta$ mean } & \multirow[b]{2}{*}{$\beta$ median } & \multirow[b]{2}{*}{$\beta$ range } & \multirow[b]{2}{*}{$\beta$ SD } & \multicolumn{6}{|c|}{ Tests for normality } \\
\hline & & & & & & $\begin{array}{l}\text { Wilk-Shapiro } \\
\text { or D'Agostino* } \\
\text { statistic }\end{array}$ & $P$ & Skew & $P$ & $\begin{array}{l}\text { Kolmogorov- } \\
\text { Smirnov } \\
\text { statistic }\end{array}$ & $P$ \\
\hline \multicolumn{12}{|l|}{$141 d$} \\
\hline All & 52 & 1.8 & 1.3 & $0.6-6.7$ & 1.4 & $0.24^{*}$ & $<0.01$ & 1.83 & $<0.05$ & 0.22 & $<0.01$ \\
\hline$\beta \leq 2.5$ & 42 & 1.2 & 1.2 & $0.6-2.3$ & 0.5 & 0.94 & $>0.05$ & 0.56 & $<0.05$ & 0.11 & $>0.05$ \\
\hline$\beta>2.5$ & 10 & 4.2 & 3.8 & $3.0-6.7$ & 1.2 & 0.88 & $>0.05$ & 1.19 & $<0.05$ & 0.15 & $>0.05$ \\
\hline \multicolumn{12}{|l|}{$143 d$} \\
\hline All & 50 & 6.0 & 3.7 & $1.4-20.1$ & 5.1 & 0.79 & $<0.01$ & 1.40 & $<0.05$ & 0.20 & $<0.02$ \\
\hline$\beta \leq 13$ & 43 & 4.3 & 3.3 & $1.4-11.7$ & 2.7 & 0.83 & $<0.01$ & 1.27 & $<0.05$ & 0.20 & $>0.05$ \\
\hline$\beta>13$ & 7 & 16.5 & 15.7 & $13.7-20.1$ & 2.5 & 0.87 & $>0.05$ & NA & & 0.15 & $>0.05$ \\
\hline \multicolumn{12}{|l|}{$151 \mathrm{~d}$} \\
\hline All & 59 & 12.0 & 7.5 & $0.0-32.5$ & 11.0 & $0.27^{*}$ & $>0.05$ & 0.63 & $<0.05$ & 0.19 & $<0.05$ \\
\hline$\beta \leq 20$ & 41 & 5.3 & 3.7 & $0.0-17.2$ & 4.5 & 0.87 & $<0.01$ & 1.07 & $<0.05$ & 0.15 & $>0.05$ \\
\hline$\beta>20$ & 18 & 27.2 & 26.4 & $21.3-32.5$ & 3.3 & 0.95 & $>0.05$ & -0.14 & $>0.05$ & 0.13 & $>0.05$ \\
\hline \multicolumn{12}{|l|}{$153 \mathrm{~d}$} \\
\hline All & 26 & 5.5 & 3.4 & $1.1-18.2$ & 4.7 & 0.77 & $<0.01$ & 1.56 & $<0.05$ & 0.24 & $>0.05$ \\
\hline$\beta \leq 10$ & 22 & 3.7 & 3.2 & $1.1-9.7$ & 2.2 & 0.86 & $<0.01$ & 1.46 & $<0.05$ & 0.21 & $>0.05$ \\
\hline$\beta>10$ & 4 & 15.1 & 15.1 & $11.9-18.2$ & 2.6 & 0.98 & $>0.05$ & NA & & 0.09 & $>0.05$ \\
\hline
\end{tabular}

NA, not applicable due to small $n$. * Where $n>50$, D'Agostino's test was used. 
continuous clock model of the switch. We reach this conclusion because analysis of large numbers of individual erythroid colonies at midswitch provides evidence for a skewed, discontinuous distribution. The patterns of $\gamma$-synthesis in fetal studies described in this report and in our published studies of newborn humans $(10,11)$ closely resemble the patterns seen in artificial mixtures of clearly discrete populations of fetal and maternal progenitors.

Further studies must involve purification of fetal and adult erythroid progenitor cells $(28,29)$, demonstration of their simultaneous presence in the fetus at midswitch and, if possible, sufficient amplification of these cells to examine their chromatinDNA relationships.

\section{Acknowledgements}

We are grateful to the staff at the New England Regional Primate Research Center for their cooperation and assistance, and we thank Drs. Jeffrey Lipton and Paul Berk for their help.

This work was supported by grants from the National Institutes of Health (HL-26132, HD-15892, AM-30141, RR-00168, HL-32262), and an Irma T. Hirschl Trust Career Scientist Award to Dr. Alter.

\section{References}

1. Stamatoyannopoulos, G., T. Papayannopoulou, M. Brice, S. Kurachi, B. Nakamoto, G. Lim, and M. Farquhar. 1981. Cell biology of hemoglobin switching. I. The switch from fetal to adult hemoglobin formation during ontogeny. In Hemoglobins in Development and Differentiation. G. Stamatoyannopoulos and A. Nienhuis, editors. Alan R. Liss, Inc., New York. 287-305.

2. Linch, D. C., L. J. Knott, C. H. Rodeck, and E. R. Huehns. 1982. Studies of circulating hemopoietic progenitor cells in human fetal blood. Blood. 59:976-979.

3. Papayannopoulou, T., S. Kurachi, B. Nakamoto, E. D. Zanjani, and G. Stamatoyannopoulos. 1982. Hemoglobin switching in culture: evidence for a humoral factor that induces switching in adult and neonatal but not fetal erythroid cells. Proc. Natl. Acad. Sci. USA. 79:6579-6583.

4. Bard, H. 1975. The postnatal decline of hemoglobin F synthesis in normal full-term infants. J. Clin. Invest. 55:395-398.

5. Alter, B. P. 1978. Beta-thalassaemia trait: imprecision of diagnosis at birth. Br. J. Haematol. 38:323-327.

6. Kidoguchi, K., M. Ogawa, J. D. Karam, J. S. McNeil, and M. S. Fitch. 1979. Hemoglobin biosynthesis in individual bursts in culture: studies of human umbilical cord blood. Blood. 53:519-522.

7. Terasawa, T., and M. Ogawa. 1980. Hemoglobin biosynthesis in individual bursts from human adult peripheral and umbilical cord blood: analysis of the relative rates of synthesis of ${ }^{\mathrm{G}} \boldsymbol{\gamma}$ and ${ }^{\mathrm{A}} \gamma$ globin chains. $J$. Cell. Physiol. 105:483-488.

8. Papayannopoulou, T., S. Kurachi, M. Brice, B. Nakamoto, and G. Stamatoyannopoulos. 1981. Asynchronous synthesis of $\mathrm{HbF}$ and $\mathrm{HbA}$ during erythroblast maturation. II. Studies of ${ }^{\mathbf{G}} \gamma,{ }^{\mathbf{A}} \gamma$, and $\beta$ chain synthesis in individual erythroid clones from neonatal and adult BFU-E cultures. Blood. 57:531-536.

9. Dean, A., A. N. Schechter, T. Papayannopoulou, and G. Stamatoyannopoulos. 1981. Heterogeneity of erythroid precursor cells. Hemoglobin quantitation in single clones by radioimmunoassay. J. Biol. Chem. 256:2447-2453.

10. Weinberg, R. S., J. D. Goldberg, J. M. Schofield, A. L. Lenes, R. Styczynski, and B. P. Alter. 1983. Switch from fetal to adult hemoglobin is associated with a change in progenitor cell population. J. Clin. Invest. 71:785-794.

11. Alter, B. P., R. S. Weinberg, J. D. Goldberg, B. T. Jackson, G. J. Piasecki, J. M. Lipton, and D. G. Nathan. 1983. Evidence for a clonal model for hemoglobin switching. In Globin Gene Expression and
Hematopoietic Differentiation. G. Stamatoyannopoulos and A. Nienhuis, editors. Alan R. Liss, Inc., New York. 431-442.

12. Alter, B. P., B. T. Jackson, J. M. Lipton, G. J. Piasecki, P. L. Jackson, M. Kudisch, and D. G. Nathan. 1981. Control of the simian fetal hemoglobin switch at the progenitor cell level. J. Clin. Invest. 67: 458-466.

13. Alter, B. P., B. T. Jackson, J. M. Lipton, G. J. Piasecki, P. L. Jackson, M. Kudisch, and D. G. Nathan. 1981. Three classes of erythroid progenitors that regulate hemoglobin synthesis during ontogeny in the primate. In Hemoglobins in Development and Differentiation. G. Stamatoyannopoulos and A. Nienhuis, editors. Alan R. Liss, Inc., New York. 331-340.

14. Alter, B. P. 1982. Regulation of hemoglobin switching during ontogeny. In Advances in Red Cell Biology. D. J. Weatherall, G. Fiorelli, and S. Gorini, editors. Raven Press, New York. 225-236.

15. Alter, B. P., and D. G. Nathan. 1982. A cellular model for hemoglobin switching. In Thalassemia: Recent Advances in Detection and Treatment. A. Cao, U. Carcassi, and P. T. Rowley, editors. Birth Defects: Original Article Series, Vol. 18, No. 7. Alan R. Liss, Inc., New York. 111-116.

16. Macklis, ?. M., J. Javid, J. M. Lipton, M. Kudisch, P. K. Pettis, and D. G. Nathan. 1982. Synthesis of hemoglobin F in adult simian erythroid progenitor-derived colonies. J. Clin. Invest. 70:752-761.

17. Friedman, A. D., D. C. Linch, B. Miller, J. M. Lipton, J. Javid, and D. G. Nathan. 1985. Determination of the hemoglobin F program in human progenitor-derived erythroid cells. J. Clin. Invest. 75:13591368.

18. Ekert, H., W. M. Ellis, K. D. Waters, and G. P. Tauro. 1982. Autologous bone marrow rescue in the treatment of advanced tumors of childhood. Cancer. 49:603-609.

19. Zar, J. H. 1974. Biostatistical Analysis. Prentice-Hall, Inc., Englewood Cliffs, NJ. 1-620.

20. Sokal, R. R., and F. J. Rohlf. 1969. Biometry. W. H. Freeman and Co., San Francisco. 1-776.

21. Zanjani, E. D., G. Lim, P. B. McGlave, J. F. Clapp, L. I. Mann, T. H. Norwood, and G. Stamatoyannopoulos. 1982. Adult haematopoietic cells transplanted to sheep fetuses continue to produce adult globins. Nature (Lond.). 295:244-246.

22. Zanjani, E. D., P. B. McGlave, A. Bhakthavathsalan, and G. Stamatoyannopoulos. 1979. Sheep fetal haematopoietic cells produce adult haemoglobin when transplanted in the adult animal. Nature (Lond.). 280:495-496.

23. Bunch, C., W. G. Wood, D. J. Weatherall, J. S. Robinson, and M. J. Corp. 1981. Haemoglobin synthesis by fetal erythroid cells in an adult environment. Br. J. Haematol. 49:325-336.

24. Wood, W. G., C. Bunch, S. Kelly, Y. Gunn, and G. Breckon. 1985. Control of haemoglobin switching by a developmental clock? $\mathrm{Na}$ ture (Lond.). 313:320-323.

25. Delfini, C., G. Saglio, U. Mazza, P. Puretto, A. Filippetti, and G. Lucarelli. 1983. Fetal haemoglobin synthesis following fetal liver transplantation in man. Br. J. Haematol. 55:609-614.

26. Papayannopoulou, T., B. Nakamoto, F. Agostinelli, G. Lucarelli, and G. Stamatoyannopoulos. 1984. Fetal to adult hemopoietic cell transplantation in man: insights to hemoglobin switching. Blood. 64(Suppl. 1):63A. (Abstr.)

27. Bleiberg, I., and M. Feldman. 1969. On the regulation of hemopoietic spleen colonies produced by embryonic and adult cells. Dev. Biol. 19:566-580.

28. Emerson, S. G., C. A. Sieff, S. C. Clark, E. A. Wang, G. G. Wong, and D. G. Nathan. 1985. Purification of fetal hematopoietic progenitors and demonstration of recombinant multipotential colony stimulating activity. J. Clin. Invest. 76:1286-1290.

29. Sieff, C. A., S. G. Emerson, A. Mufson, T. G. Gesner, and D. G. Nathan. 1986. The dependence of highly enriched human bone marrow progenitors on hematopoietic growth factors and their response to recombinant erythropoietin. J. Clin. Invest. 77:74-81. 